Bailey (University of Manchester), and Jack Hills (Los Alamos) showed that such an orbit was unstable and would be likely to escape from the Solar System in less than $1.0 \mathrm{Gyr}$. Since capture of a companion star is extremely unlikely, the orbit must have been more tightly bound in the past.

The Berkeley group believes that the last periodic extinction was about 12-14 Myr ago, so the proposed companion star, which they call Nemesis, is now near aphelion. Muller described an optical survey of 5,000 low-mass red dwarf stars, which is expected to examine all candidate stars visible from the Northern Hemisphere by the end of this year, and will be extended to the Southern Hemisphere in 1986. Nemesis, if it exists, might also be detected by the all-sky survey of the Extreme Ultraviolet Explorer Satellite, scheduled to be launched in late 1988 .

Whitmire and John Matese (University of Southwestern Louisiana) suggested an alternative mechanism for creating periodic cometary showers that involves a tenth planet, some 100-150 AU from the Sun. They suggest that the orbit of the planet is eccentric and highly inclined, and that it has swept a 'clear zone' out of the inner ring of the Oort cometary cloud. As the orbit precesses, however, it will occasionally come close to the edges of the clear zone and again perturb the comets there. The comets are first thrown into Jupitercrossing orbits, and then some fraction of them reach the Earth's orbit. Comets which do not hit the Earth are eliminated by Jupiter's perturbations in a period of about 1 Myr. Whitmire and Matese believe that the tenth planet has a mass one and a half times that of the Earth and an orbit inclination of at least 45 degrees.

I raised a number of objections to the hypotheses involving cometary showers. Current estimates of the cratering rate on the Earth and Moon from known craters on dated surfaces agree well with expectations from the observed flux of Earthcrossing comets and asteroids, whereas adding intense comet showers every $26 \mathrm{Myr}$ would raise the cratering rate by a factor of 5-18, inconsistent with the observed craters. The only way out of this dilemma is to assume that the Solar System is currently in the midst of a cometary shower, and that the 'steady state' observed cometary and asteroid flux (many dynamicists hold that the Earth-crossing asteroids are extinct comets) is anomalously high. This might work with the 31-33 Myr period, which puts the last periodic extinction only 4 - 5 Myr ago, but not with the $26 \mathrm{Myr}$ period, which sets the last extinction some 10 Myr farther back in time. Moreover, analyses of impact melts from terrestrial craters suggest that many were made by highly differentiated meteorites, like irons and achondrites (similar to basalts). This is inconsistent with a cometary source, since comets are believed to be the most pristine undifferentiated objects in the Solar System, a conclusion supported by analyses of interplanetary dust collected by highflying aircraft.

The advocates of cometary showers countered that the identifications of the objects that formed the crater are still very tentative, and that the flux of comets in a shower might be up to an order of magnitude less than their original estimates, thus lowering the predicted cratering to acceptable levels. The question of whether the lower fluxes would also decrease the probability of major biological extinctions was not answered.

In the end, no hypothesis for or against periodicity emerged as clear winner, and few converts were made, but a very convincing picture emerged of the ways in which the Galaxy could affect the environment within the Solar System and on Earth. Although the conference participants did not come to any clear concensus on the extinction problem, they returned home with broadened horizons and, perhaps, with the temptation to continue to explore beyond their specialized holes.

Paul Weissman is in the Earth and Space Sciences Division, Jet Propulsion Laboratory, California Institute of Technology, Pasadena, California 91109. USA.

\title{
Biogeology Sulphur-eating tube worms take to the Oregon breaches
}

\author{
from Roger N. Anderson
}

SCIENTISTS attending meetings of the American Geophysical Union (AGU) have grown accustomed to new reports of undersea oases of brilliant red tube worms and giant red-meated clams clustered around black smokers, which billow forth $400^{\circ} \mathrm{C}$ hydrothermal fluids at the axes of midocean ridges. New communities from the eastern Pacific have been reported with remarkable regularity since their initial discovery in 1977. More were reported at the AGU winter meetings in San Francisco (4-9 December 1984), but there was a big surprise in store. A series of four consecutive papers ${ }^{1-4}$ documented the presence of sulphur-eating tube worms and giant clams in a completely new environment: on the accretionary pile of sediments found above a subduction zone, where there are no hot springs.

The biological communities were found living along 'breaches' or open rifts that were discovered during dives of the submersible $A$ lvin along a series of anticlinal folds of newly accreted sediments on the continental margin off Oregon, in the north-west United States. No hot-water springs were detected by the sensitive thermometers on Alvin, but methane-enriched waters were captured. These, along with methanederived calcium carbonate cementation found within the surface sediments, verify that sulphur-rich waters are being expelled along the breaches. Methane and sulphur are both added to hydrothermal waters during chemical interaction with basalts of the oceanic crust.

Though not associated with hot springs, the methane-rich waters of the breaches probably have a source similar to that at ridge crests. The subduction of the Juan de Fuca plate under the coast of Oregon is the likely source of the sulphur and methane. Waters circulating in the crust eventually end up as pore fluids in sediments being accreted to the west coast of Oregon. Dewatering of these sediments is apparently a more dominant physical process than previously thought, since sufficient flow must be occuring to supply enough nutrients to support the same kind of biological community as that living near ridge-axis black smokers. Contrary to popular belief, these new observations show that hot temperatures are not required to support the organisms.

The typical sedimentary diagenesis that is observed in the area points to widespread venting all along this margin. The microcrystalline dolomite, magnesian calcite, and aragonite that surround the vents result from methane reactions. Such mineralogy is common throughout older accretionary complexes of the world. Dewatering of 20 per cent by volume of the accretionary sediments is required to support the volume of diagenetic sediments observed off Oregon. The dewatering becomes more pronounced as one proceeds away from the trench axis toward the shoreline. Tubeworm communities were sampled on vents in sediments accreted up to 1.8 million years ago.

These discoveries, and another report ${ }^{5}$ of similar sulphur-eating organisms at the foot of the Florida escarpment, where sulphide-rich hypersaline waters are seeping out at ambient temperatures onto the sea floor $3 \mathrm{~km}$ below the surface, show that our tube-worm friends are more plentiful than ever anticipated. The joy of all this for geologists is that we can learn much about subsurface hydrological processes while observing some of nature's true wonders.

1. Carson, B., Ritger, S.D. \& Suess, E. EOS 65, 1089 (1984). 2. Kulm. L.D., Thornburg, T.M. \& Carson, B. EOS 65,1090 (1984).

3. Moore, J.C., Kulm, L.D., Lewis, B.T.R. \& Cochran, G. B. Moore, J.C., Kul

EOS 65, 1089 (1984).
4. Suess, E. \& Mossoth, G.D. EOS 65, 1089 (1984).

5. Paull, C.K. et al. Science 226, 965 (1984).

Roger N. Anderson is at the Lamont-Doherty Geological Observatory, Columbia University, Palisades, New York 10964, USA. 